Retrospective Evaluation

\title{
Percutaneous Sacroplasty for Non-neoplastic Osteoporotic Sacral Insufficiency Fractures
}

\author{
Dong-Hwa Heo, MD, PhD and Choon-Keun Park, MD, PhD
}

From: Department of Neurosurgery, Spine Center, The Leon Wiltse Memorial Hospital

Gyeonggi-do, Korea

Address Correspondence: Dong Hwa Heo. MD, PhD Department of Neurosurgery,

The Leon Wiltse Memorial Hospital, 994-3, Ingye-dong, Paldal-gu, Suwon-si, Gyeonggi-do, Korea (442-833) E-mail:

spinesurgery@naver.com

Disclaimer: There was no external funding in the preparation of this manuscript. Conflict of interest: Each author certifies that he or she, or a member of his or her immediate family, has no commercial association (i.e., consultancies, stock ownership, equity interest, patent/licensing arrangements, etc.) that might pose a conflict of

interest in connection with the submitted manuscript.

Manuscript received: 06-22-2016 Revised manuscript received: 08-08-2016

Accepted for publication: o8-12-2016

Free full manuscript: www.pain physicianjournal.com
Background: Osteoporosis is an important problem in those of advanced age. Osteoporosis can induce sacral insufficiency fractures (SIFs). As average life expectancy increases, the number of patients with osteoporotic SIFs also increases. Osteoporotic SIFs cause severe low back pain and immobilization. Treatment of SIFs varies from analgesia to surgery.

Objectives: To describe our experience and assess the safety and effectiveness of minimally invasive percutaneous sacroplasty in patients with osteoporotic SIFs.

Study Design: Retrospective evaluation.

Setting: The spine and joint specialized hospital's research center.

Methods: We reviewed cases of percutaneous sacroplasty performed since 2009. We used data only from patients with osteoporotic SIFs who were followed for more than 12 months after sacroplasty. Tumor-related SIFs were excluded from our analysis. The following clinical parameters were investigated: initial diagnosis, symptoms, visual analog scale (VAS) of pain, functional mobility scale (FDC) score, past history of illness, amount of bone cement infused, and complications related to sacroplasty. Also, the following radiological parameters were analyzed: the pattern of SIFs, T-score cement leakage, and concomitant fractures in other sites.

Results: Sixty-eight patients were enrolled in our study (4 men and 64 women). The mean age of the patients was $76.8 \pm 6.2$ years. All patients had severe osteoporosis (mean T score: $-3.9 \pm 0.5$ ). Percutaneous sacroplasty was performed under fluoroscopic guidance. No major complications or procedure-related morbidity occurred. FDS and VAS scores significantly improved after sacroplasty, and the improvements lasted through the final follow-up period $(P<0.05)$

Limitations: This study is a retrospective analysis without a control group.

Conclusion: Percutaneous sacroplasty is an effective minimally invasive treatment for osteoporotic SIFs refractory to conservative management. The study patients experienced significant relief of pain and increased mobility.

Key words: Sacrum, sacroplasty, insufficiency fractures, osteoporosis, minimally invasive surgery

IBR Number: 2016-W03

Pain Physician 2017; 20:89-94

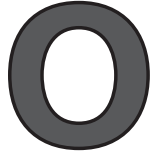
steoporosis and tumors can lead to sacral insufficiency fractures (SIFs) (1-5). Recently, the number of geriatric patients with osteoporosis has increased. Osteoporosis is the main cause of SIFs $(5,6)$. SIFs cause severe low back pain, buttock pain, groin pain, and immobilization $(4,5,7,8)$. Some patients become bedridden due to severe pain caused by SIFs (7). The symptoms of SIFs are similar to those of degenerative lumbar diseases such as spinal stenosis. Moreover, sagittal and axial 
images taken with lumbar magnetic resonance imaging (MRI) may not reveal the presence of SIFs (7). Clinicians may overlook SIFs, which leads to delayed diagnoses or misdiagnoses. Current treatment recommendations vary from analgesia to surgery $(4,9)$. The first-line treatment for SIFs is conservative management including bed rest and analgesic medication. However, long-term immobilization due to SIFs increases the risk of deep vein thrombosis, pulmonary embolism, and pneumonia $(5,7)$.

Pelvic insufficiency fractures, including sacral fractures, result in morbidity and mortality that is related to immobilization (overall one-year mortality rate: $~ 14 \%$ ) (8). Optimal diagnosis and treatment of SIFs in geriatric patients is necessary to prevent morbidity and mortality. Percutaneous sacroplasty using polymethylmethacrylate (PMMA) is a minimally invasive treatment for SIFs that can improve pain and functional status; it includes vertebroplasty and kyphoplasty $(1,4,10)$.

Osteoporosis is an important problem in those of advanced age. As average life expectancy increases, the number of patients with osteoporotic SIFs also increases. Previous studies have not investigated the clinical outcomes of percutaneous sacroplasty specifically for osteoporotic SIFs. The purpose of our study is to describe our experience and to assess the safety and effectiveness of minimally invasive percutaneous sacroplasty in patients with osteoporotic SIFs.

\section{Methods}

Only patients with osteoporotic SIF were enrolled in this study. Those with metastatic lesions or primary bone tumors in the sacrum were excluded. Osteoporotic SIF was diagnosed by MRI examination. If SIF was suspected from routine sagittal and axial MR images, the coronal section of short tau inversion recovery (STIR) or T2-weighted images with fat suppression were evaluated (Fig. 1). If MRI findings appeared to show primary bone tumors or metastasis in the sacrum, and if patients had a history of cancer treatment or pelvic irradiation, gadolinium-enhanced MRI and positron emission tomography (PET) scans were performed for the evaluation of primary or metastatic tumors.

All patients were initially treated with conservative management including analgesic medications and bed rest. If patients presented with severe refractory pain or non-ambulatory status (functional mobility scale $>3$, Table 1 ) even though conservative management was used for at least 3 weeks, we performed percutaneous sacroplasty under local or epidural anesthesia. We used low pressure-high viscosity sacroplasty with PMMA via the short axis approach under fluoroscopic guidance (Fig. 2) (1). Our target of augmentation was the sacral ala (6). If the SIF was not found in the S1 segment, we did not perform percutaneous sacroplasty.

Clinical parameters (symptoms, signs, initial diagno-
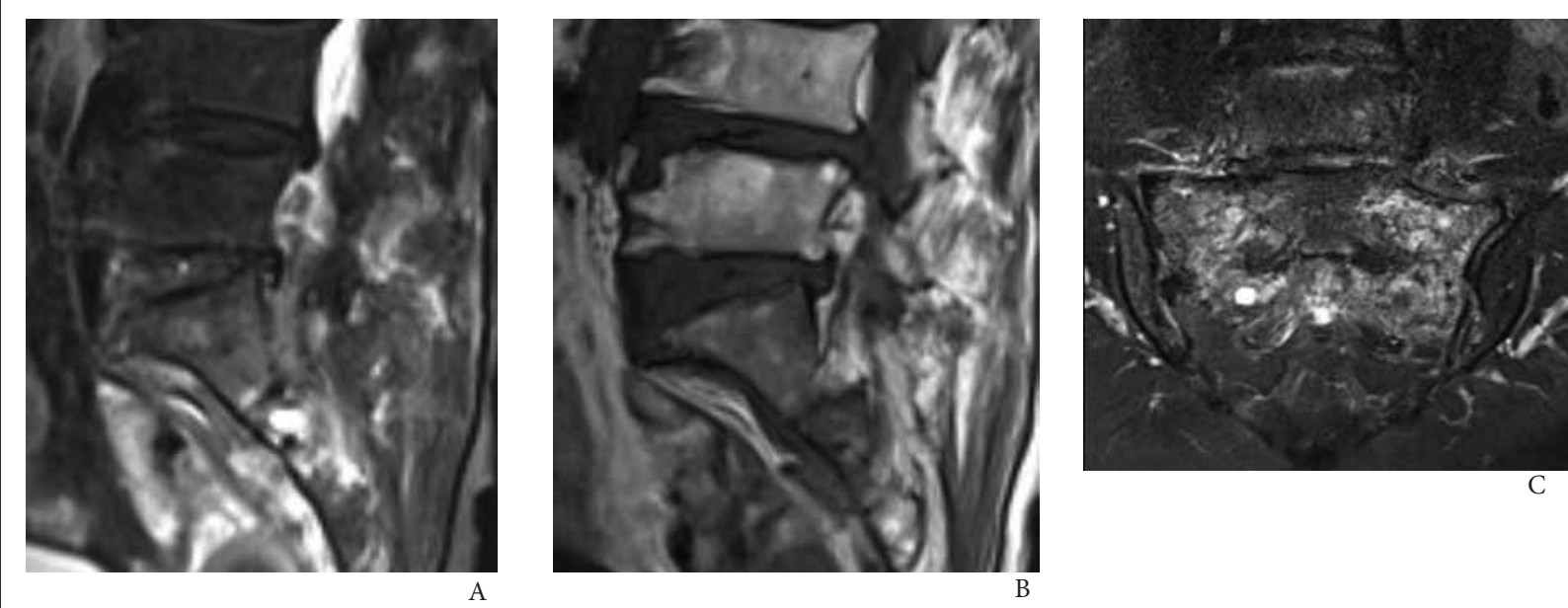

Fig. 1. MRI of a 71-year-old woman with severe buttock and groin pain. MR sagittal STIR (A) and T1 weighted (B) images show marrow edema in the S1 and surrounding area. An MR coronal STIR image depicts bilateral sacral insufficiency fractures $(C)$. 
sis, visual analog scale (VAS) score, functional mobility scale (FDC) score (7), past history of illness, amount of bone cement (PMMA) infused, and complications related to sacroplasty) were investigated. Also, radiological parameters ( $\mathrm{T}$ score, cement leakage seen on postoperative x-ray, and concomitant fractures) were analyzed.

We performed this investigation in accordance with our institutional guidelines, which comply with international laws and policies (Institutional Review Board of the Leon Wiltse Memorial Hospital, \#2016-W03).

\section{Statistical Analysis}

Statistical analysis was assessed with the Mann Whitney $U$ test and the Wilcoxon rank sum test. $P<$ 0.05 was considered statistically significant. $\mathrm{R}$ (version 3.2) was used for the statistical analysis.
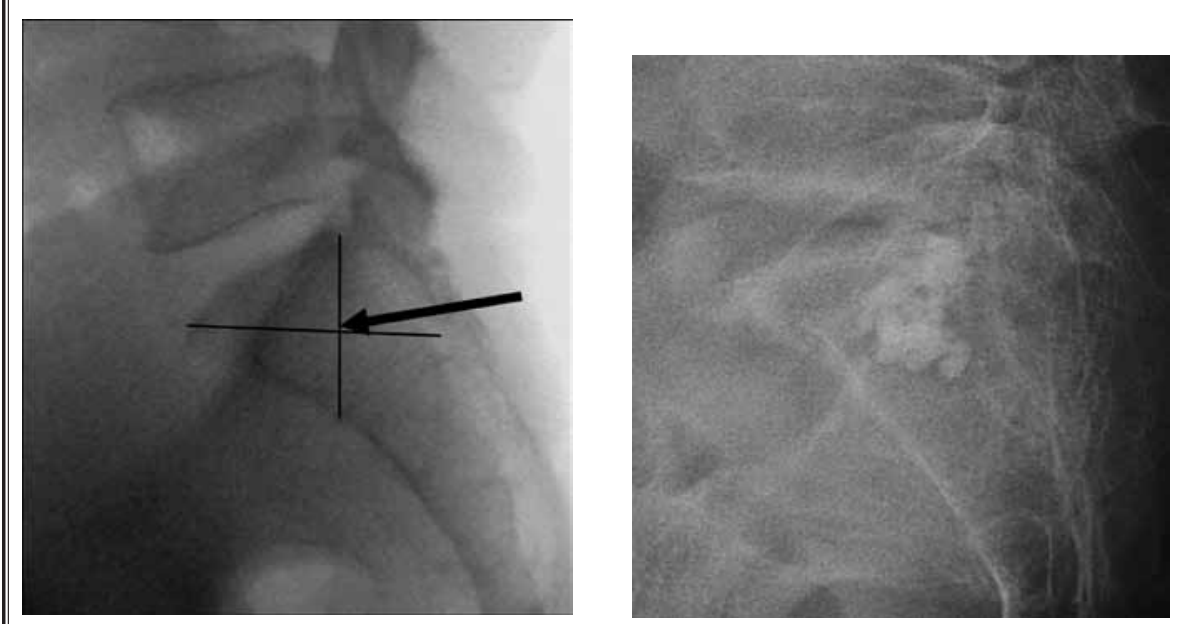

A

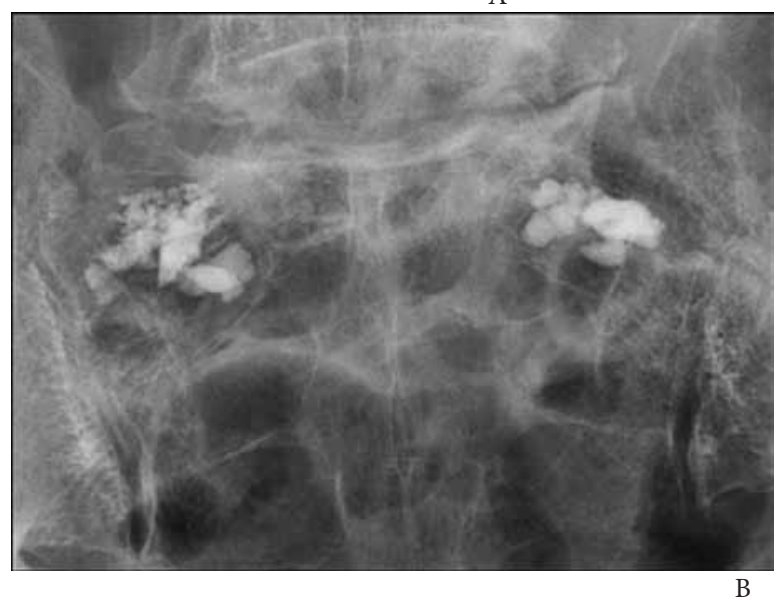

\section{Results}

Fig. 2. The fluoroscopically guided short axis approach used to perform percutaneous sacroplasty (A). A postoperative radiograph (B and $C$ ).

We have performed percutaneous sacroplasty on 79 patients with osteoporotic SIFs since March 2009. A total of 68 patients were enrolled in our study (4 men and 64 women). All enrolled patients were followed up for more than 12 months after sacroplasty (mean follow up period: $15.86 \pm 5.69$ months). The mean age of the patients was $76.8 \pm 6.2$ years. All patients had severe osteoporosis (mean T score: $-3.9 \pm 0.5$ ). The etiology of the fractures was osteoporotic insufficiency (36 cases) and osteoporosis with minor trauma (32 cases). Four patients had secondary osteoporosis due to corticosteroid medications and 2 patients had a history of pelvic irradiation to treat gynecologic tumors without sacral metastasis (Table 2).
Table 1. Functional mobility scale.

\begin{tabular}{|c|l|}
\hline Score & \multicolumn{1}{|c|}{ Functional mobility scale } \\
\hline 0 & Full acitivity \\
\hline 1 & Walking with assistance \\
\hline 2 & Walking with assistance for short periods \\
\hline 3 & $\begin{array}{l}\text { Walking with assistance for activities of daily living/ } \\
\text { appointments only }\end{array}$ \\
\hline 4 & Confined to a wheelchair \\
\hline 5 & Bedridden \\
\hline
\end{tabular}


The symptoms of SIFs were back pain, buttock pain, radiating leg pain, groin pain, coccyx area pain, and complete bedridden status (Table 3 ). A positive finding on Patrick's test was detected in 5 patients, and limitation of straight leg raising was seen in 7 patients. Initial misdiagnoses by clinicians in our hospital or other hospitals included spinal stenosis, herniated lumbar discs, hip joint problems, and thoracolumbar compression fractures (Table 4). Nineteen patients had concomitant

Table 2. Patient characteristics.

\begin{tabular}{|l|c|}
\hline \multicolumn{1}{|c|}{ Characteristic } & Value \\
\hline Gender (male/female) & $4 / 64$ \\
\hline Age (years) & $76.8 \pm 6.2$ \\
\hline T-score & $-3.9 \pm 0.5$ \\
\hline Mean follow up periods (months) & $15.86 \pm 5.69$ \\
\hline Fracture etiology & \\
Osteoporotic insufficiency & 36 \\
Osteoporosis with minor trauma & 32 \\
\hline Specific illness history & \\
Secondary osteoporosis & 4 \\
Pelvis irradiation & 2 \\
\hline Type & 47 \\
Bilateral fracture & 21 \\
\hline Unilateral fracture & \\
\hline Concomitant fractures & 15 \\
Thoracolumbar fracture & $1.61 \pm 0.43$ \\
\hline Pelvic bone fracture & \\
\hline Bone cement injection amount at one side (ml) & \\
\hline
\end{tabular}

Table 3. Symptoms associated with sacral insufficiency fractures.

\begin{tabular}{|l|c|}
\hline \multicolumn{1}{|c|}{ Symptoms } & Percentage \\
\hline Buttock pain & $47 / 68(69.1 \%)$ \\
\hline Lower back pain & $35 / 68(51.5 \%)$ \\
\hline Radiating leg pain & $33 / 68(48.5 \%)$ \\
\hline Groin pain & $12 / 68(17.6 \%)$ \\
\hline Bedridden status & $10 / 68(14.7 \%)$ \\
\hline Coccyx area pain & $5 / 68(7.4 \%)$ \\
\hline
\end{tabular}

Table 4. Initial misdiagnosis before correct diagnosis of sacral insufficiency fractures.

\begin{tabular}{|l|c|}
\hline \multicolumn{1}{|c|}{ Misdiagnosis } & Cases \\
\hline Spinal stenosis & 7 \\
\hline Thoracolumbar fracture & 6 \\
\hline Hip joint problem & 3 \\
\hline Herniated lumbar disc & 2 \\
\hline
\end{tabular}

fractures, 4 pelvic bone (Fig. 4) and 15 thoracolumbar compression fractures (Table 2).

FDS and VAS scores were significantly improved after sacroplasty, and the improvements lasted through the follow-up period. FDS scores significantly improved from $3.82 \pm 0.68$ preoperatively to $1.09 \pm 0.74$ at the last follow-up visit. VAS scores significantly improved from $8.65 \pm 0.97$ to $2.41 \pm 1.07$.

The mean amount of PMMA injected into one side was $1.61 \pm 0.43 \mathrm{~mL}$. No major complications or procedure-related morbidity occurred. Cement leakage into the sacroiliac joint occurred in 2 patients (Fig. 3). Fortunately, there were no symptoms related to cement leakage. There were no cases of cement leakage into the neural foramen.

\section{Discussion}

Osteoporosis influences morbidity and mortality in the elderly. Fractures related to osteoporosis include immobilization and disability. Moreover, prolonged immobilization can cause complications such as deep vein thrombosis, pneumonia, pressure sores, and muscle weakness $(5,7)$. SIFs can occur in osteoporosis patients with or without minor trauma. The sacrum is a weightbearing structure that is important for ambulation. Therefore, SIFs induce severe pain and disability, such as being bedridden.

Some patients presented with groin pain and pain provocation on flexion and abduction of the hip joint (Patrick's sign); these cases were often misdiagnosed as hip joint problems. Other patients presented with severe buttock pain and pain that radiated into the legs. These cases were subject to misdiagnosis as lumbar degenerative diseases such as lumbar stenosis (6). Lumbar decompression surgeries were recommended in some cases at other hospitals. Signs and symptoms similar to those associated with problems in the sacroiliac joint, hip joint, or lumbar disease were one of the reasons for delayed diagnoses. Optimal diagnosis and recognition of SIFs may prevent unnecessary operations such as lumbar surgeries.

In patients diagnosed with SIFs, clinicians should look for fractures in other sites such as the pelvic bone or vertebrae. Concomitant fractures may influence postoperative clinical outcomes after percutaneous sacroplasty. Some patients with concomitant pelvic bone fractures did not experience improvements following sacroplasty (Fig. 3). Whole sagittal spine MRI and bone scintigraphy are useful for evaluation of not only SIFs but also concomitant vertebral and pelvic bone fractures $(6,7)$. 


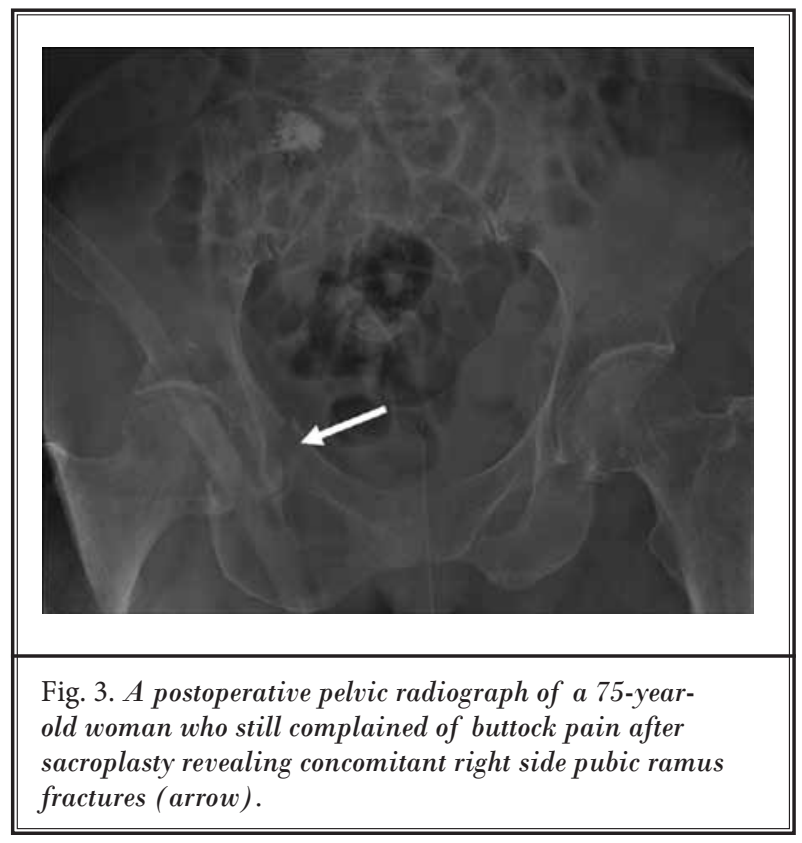

There are 2 trajectories of sacroplasty, the short axis approach and the long axis approach. The ala portion of the sacrum is important for biomechanical stabilization. We used the short axis approach and targeted the sacral ala. We injected a small volume of PMMA (about $1-2 \mathrm{~mL}$ ) into each ala, and witnessed improvements in pain and disability. The short axis approach can be performed using fluoroscopic guidance without computed tomography (CT).

PMMA cement leakage is one complication of sacroplasty $(4,6,7)$. PMMA leakage into the neural foramen is a serious complication. In such cases, surgical removal of the cement mass may be required. In our cohort, PMMA leakage to the sacroiliac joint occurred in just 2 cases (Fig. 4). Fortunately, those 2 patients did not experience any side effects related to PMMA leakage. High viscosity and low pressure injection of PMMA may help to prevent cement leakage. We used a large diameter cannula and filler which is also used for vertebroplasty or osteoplasty to perform high viscosity, low pressure injections. Some researchers used a balloon or radiofrequency probe for high viscosity PMMA injections during sacroplasty $(8,9)$. Needle or cannula location may be important for foraminal leakage of PMMA. The tip of the cannula being close to the foramen may increase the risk of foraminal PMMA leakage. In some cases, we used a venogram with contrast media, as done in vertebroplasty, to evaluate foraminal leakage (Fig. 5). If contrast media leaked into the $\mathbf{S 1}$ foramen
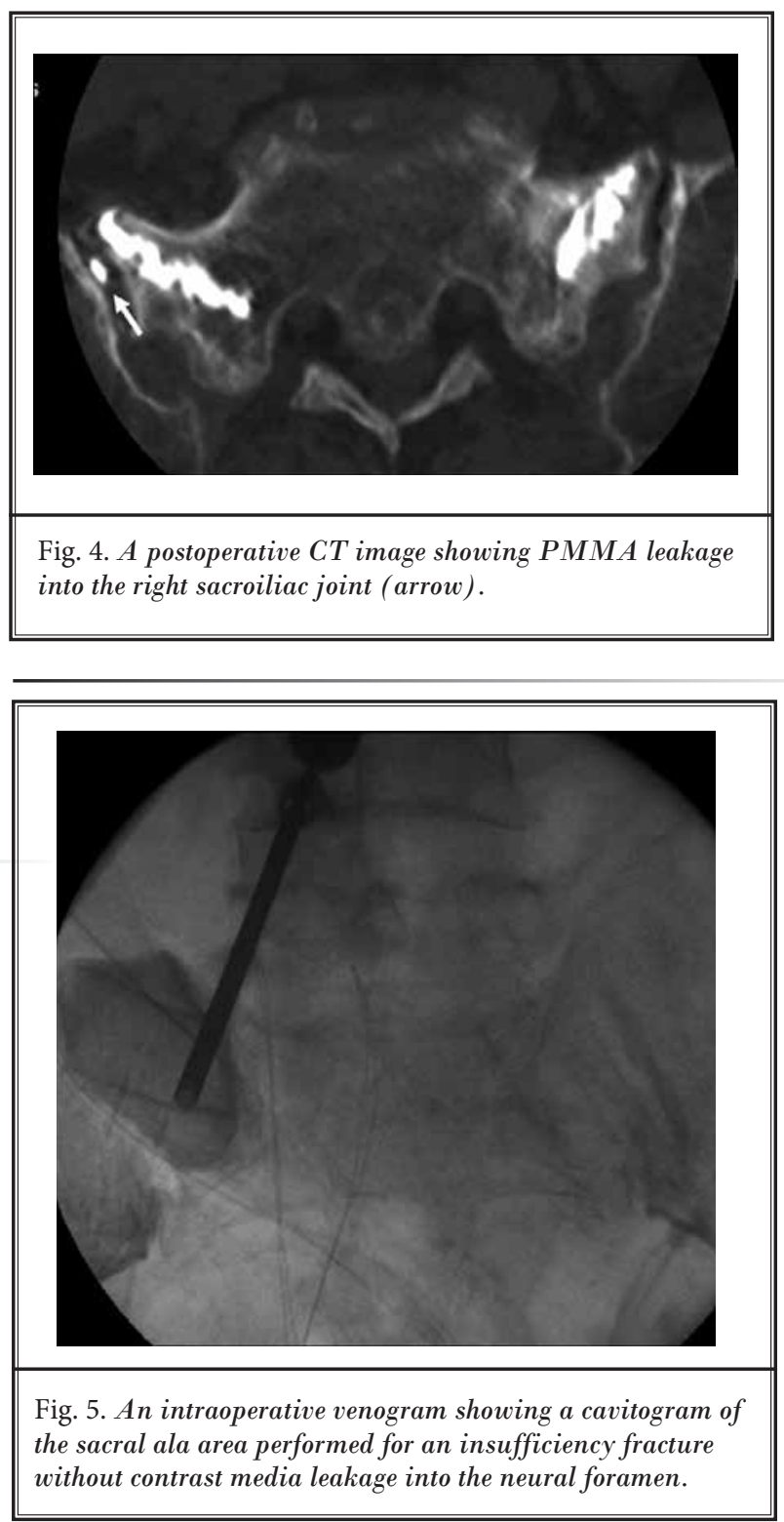

before PMMA injection, we repositioned the tip of the cannula.

Previously reported clinical outcomes of sacroplasty are favorable (4-8). Percutaneous sacroplasty can improve pain and functional status and prevent the need for sacro-pelvic fusion surgery in patients with osteoporotic or tumorous SIFs (4). In our cohort, the clinical outcomes of sacroplasty for the treatment of osteoporotic SIFs were good, as in other studies. However, there have been no randomized case controlled studies. A double-blinded randomized case controlled study should be performed to optimize the evaluation of the 
clinical results of sacroplasty. One limitation of our study is that it was not double-blinded and randomized.

\section{Conclusion}

Percutaneous sacroplasty is an effective minimally invasive treatment for osteoporotic sacral insufficiency fractures refractory to conservative management. Percutaneous sacroplasty produced significant gains in pain relief and functional status.

\section{Disclosure}

The authors have no personal, financial, or institutional interest in any of the drugs, materials, or devices described in this article.

\section{Acknowledgments}

Special thanks to Dr. Dong Chan Lee and Dr. Dong Hwan Lim.

\section{References}

1. Pereira LP, Clarencon F, Cormier E, Rose $M$, Jean B, Le Jean L, Chiras J. Safety and effectiveness of percutaneous sacroplasty: A single-centre experience in 58 consecutive patients with tumours or osteoporotic insufficient fractures treated under fluoroscopic guidance. Eur Radiol 2013; 23:2764-2772.

2. Butler $\mathrm{CL}$, Given $\mathrm{CA}$, 2nd, Michel SJ, Tibbs PA. Percutaneous sacroplasty for the treatment of sacral insufficiency fractures. AJR 2005; 184:1956-1959.

3. Trouvin AP, Alcaix D, Somon T, Zarnitsky C. Analgesic effect of sacroplasty in osteoporotic sacral fractures: A study of six cases. Joint Bone Spine 2012; 79:500-503.

4. Moussazadeh N, Laufer I, Werner T, Krol
G, Boland P, Bilsky MH, Lis E. Sacroplasty for cancer-associated insufficiency fractures. Neurosurgery 2015; 76:446-450; discussion 450.

5. Talmadge J, Smith K, Dykes T, Mittleider D. Clinical impact of sacroplasty on patient mobility. J Vasc Interv Radiol 2014; 25:911-915.

6. Onen MR, Yuvruk E, Naderi S. Reliability and effectiveness of percutaneous sacroplasty in sacral insufficiency fractures. J Clin Neurosci 2015; 22:1601-1608.

7. Gupta AC, Chandra RV, Yoo AJ, LeslieMazwi TM, Bell DL, Mehta BP, Vanderboom TL, Rabinov JD, Larvie M, Hirsch JA. Safety and effectiveness of sacroplasty: A large single-center experience.
AJNR 2014; 35:2202-2206.

8. Eichler K, Zangos S, Mack MG, Marzi I, Vogl TJ. Outcome of long-axis percutaneous sacroplasty for the treatment of sacral insufficiency fractures with a radiofrequency-induced, high-viscosity bone cement. Skeletal Radiol 2014; 43:493-498

9. Andresen R, Ludtke CW, Radmer S, Kamusella P, Schober HC. Radiofrequency sacroplasty (RFS) for the treatment of osteoporotic insufficiency fractures. Eur Spine J 2015; 24:759-763.

10. Garant M. Sacroplasty: A new treatment for sacral insufficiency fracture. ] Vasc Interv Radiol 2002; 13:1265-1267. 